\title{
SCORING PROGRAM DEVELOPMENT COURSE FOR SOFTWARE EDUCATION USING COMPUTATIONAL THINKING MODEL
}

\author{
Kyeong Hur ${ }^{1}$, Won-Sung Sohn ${ }^{1 *}$ and Kil Young Kwon ${ }^{2 \dagger}$ \\ ${ }^{1}$ Dept. of Computer Education, Gyeongin National University of Education, \\ Gyesan-Dong San 59-12, Gyodae-Gil 45, Gyeyang-Gu, Incheon, 407-753, Korea \\ ${ }^{2}$ Dept. of Family Medicine, Eulji General Hospital, Hagye-Dong 280-1, \\ Hanguelbiseok-street 68, Nowon-Gu, Seoul, 139-872, Korea \\ *sohnws@ginue.ac.kr
}

\begin{abstract}
In the age of the fourth industrial revolution, major social needs for indispensable SW (software) education are the CT (computational thinking) training. However, there is a lack of sympathetic software projects in the CT training for nonmajor students, when using a block coding tool like the scratch 3.0. Effective CT training examples should include the use of abstraction, decomposition, pattern recognition, and algorithmic thinking techniques, along with adequate structures for data storage. In this paper, we focused on how to make a model of CT processes to achieve the goals of SW education. Then, we proposed a CT model and its educational use-case in the project of developing an OX-type scoring program. The proposed OX-type scoring program was systematically developed in accordance with predefined problem-solving steps in the CT model. This proposal can be applied to various SW education subjects to educate the problem-solving steps using software and to enhance the understanding of CT processes.
\end{abstract}

Keywords - Block coding, Computational thinking, Information, Scratch 3.0, Software education

\section{INTRODUCTION}

In the SW (software) education, the CT (computational thinking) training is indispensable. Computational thinking is defined as the ability to solve problems efficiently based on computing principles. In other words, the excellence of the problemsolving process is more important than solving the problem itself [1-3]. For this computational thinking, four key thinking skills are needed: First, the decomposition ability to crack down is to break up large, complex problems or structures into small pieces. Second, pattern recognition ability is to find the similarity within each cleaved part or between cleaved parts. Third, abstraction thinking is about focusing only on the important information, ignoring the relevant details. Finally, algorithmic thinking is developing a step-by-step solution to a problem or a rule to follow to solve a problem [13].

Thus, effective CT training projects should include the use of abstraction, decomposition, pattern recognition, and algorithmic thinking techniques along with adequate structures for data storage, even in the subjects for non-major students [4-6].

Received: July 12, 2019

Reviewed: September 27, 2019

Accepted: October 1, 2019

* Corresponding Author

$\dagger$ Co-first Author

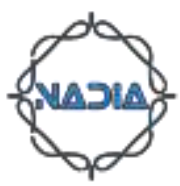


Non-major students need more examples of effective software projects for the CT training, from the case of using a block coding tool like the scratch 3.0 [5].

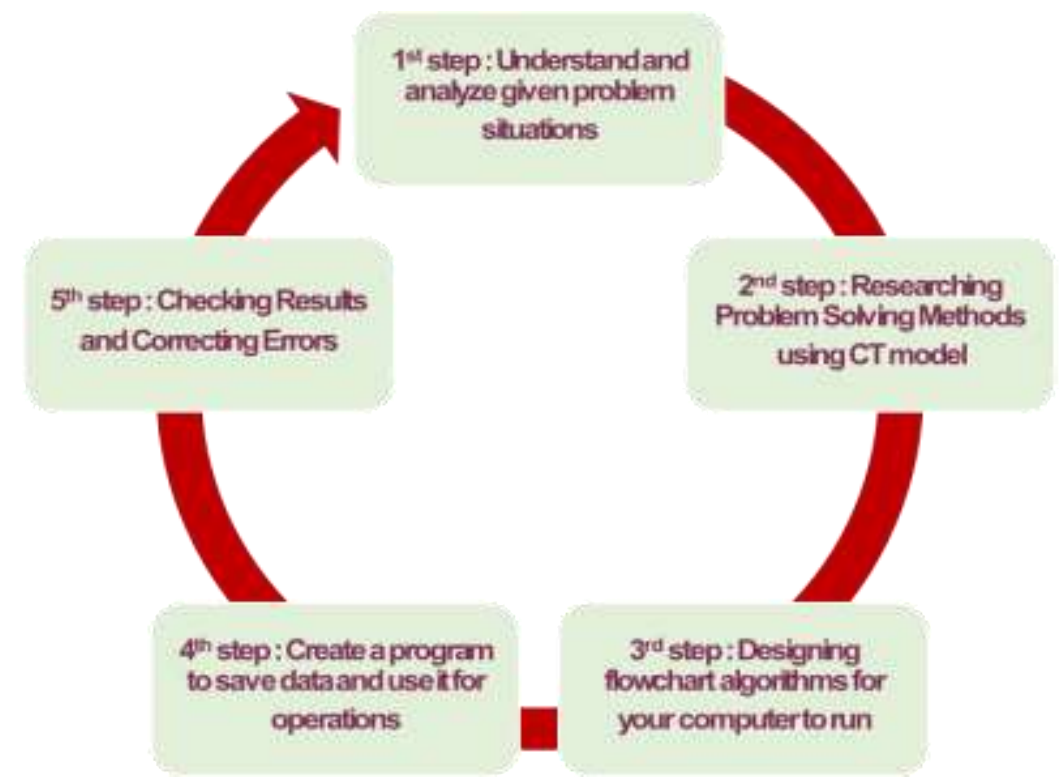

Fig. 1 SW-making steps using a CT model to solve a problem

In this paper, we defined the five SW-making steps using a CT model to solve a problem as in Figure 1 [7-10]. Furthermore, whole SW education stages including the SWmaking steps using a CT model executed in this paper are explained in Figure 2. Whole SW education stages are divided into four parts. The first A stage is 'Understanding SWmaking steps using a CT model'. The second B stage is 'Designing problem-solving algorithms using CT model'. The 'SW-making steps using a CT model' are composed of the five steps in Figure 1. The third stage is 'Development of a problem-scoring program'. The fourth stage is 'Development of own problem-scoring SW by each team'.

Objectives of this whole SW education course are defined as follows. Firstly, students understand the SW-making steps using a CT model to solve a problem. Secondly, students understand that in software development, it is necessary for students to have a high level of CT thinking ability to store and use data to mathematical operations [10-13]. Thirdly, the software should be developed to process the given data to produce useful information [7-13].

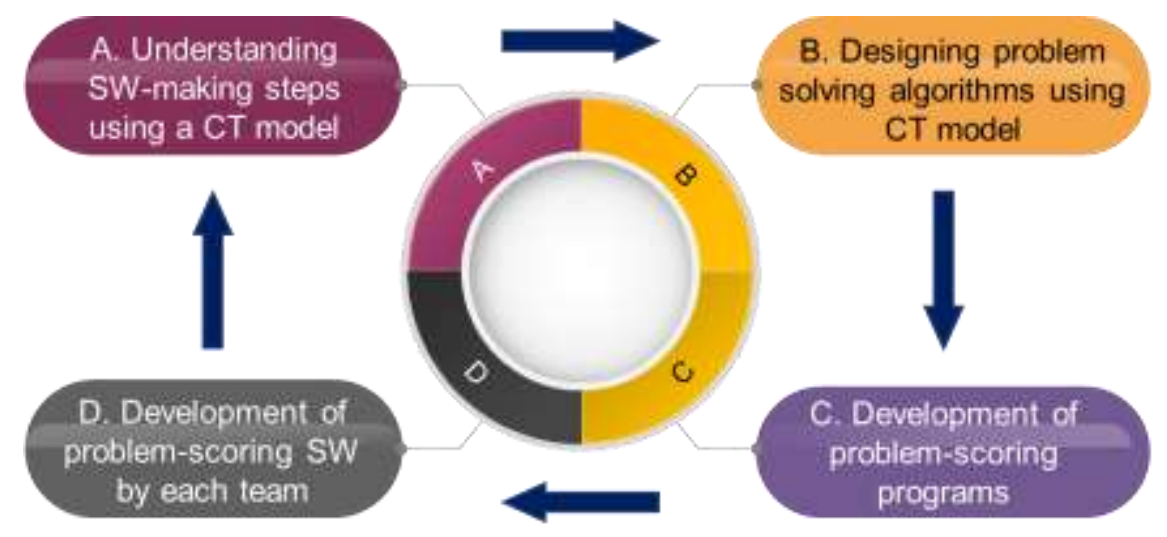

Fig. 2 Composition of whole SW education stages 
In this paper, we focused on making a model of CT processes. And to educate each problem-solving step with a CT model, we proposed a CT model and a project of developing an OX-type scoring program in the following Sections. In this project, the given problem is 'Let's create a program that automatically scores OX-type problems.' For the first step, students can analyze the given OX-type scoring problem situations. The proposed OX-type scoring program was systematically developed in accordance with predefined problem-solving steps in the CT model. From the second to the fifth SWmaking steps using the proposed CT model are explained from the third Sections.

\section{PROPOSED HIERARCHICAL COMPUTATIONAL THINKING MODEL}

In Figure 3, an abstraction thinking model is explained. This model appears in the first layer problem-solving process. Here, each Pn block must have a function of receiving a value and outputting it through a judgment process. Knowing the inner functions in the Pn blocks and designing the first layer problem-solving process, it shows student's abstraction thinking ability and problem-decomposition ability.

In the second layer problem-solving process for P1 and P2 blocks, there are Mn blocks. This Mn block is a function execution block without judgment process. A function execution block denoted by $\mathrm{Mn}$ is defined by collecting $\mathrm{Fn}$ codes that perform corresponding low-level roles in a program. Using Mn functions can reduce duplicate codes and reuse of already created codes. These Pn blocks are made of Mn function blocks. Also, while analyzing the Pn blocks, we find and construct Mn function blocks that are commonly used through pattern recognition.

In Figure 4, these Mn blocks are made of Fn blocks which are the lowest layer functions. Also, while analyzing the Mn blocks, we find and construct Fn blocks that are commonly used through pattern recognition. In this order, creating top-level Pn blocks to bottom-most Fn block groups represents a top-down algorithmic thinking process. We proposed this computational thinking model and its educational use-case in the task of developing an OX-type scoring program using the scratch 3.0 [5].

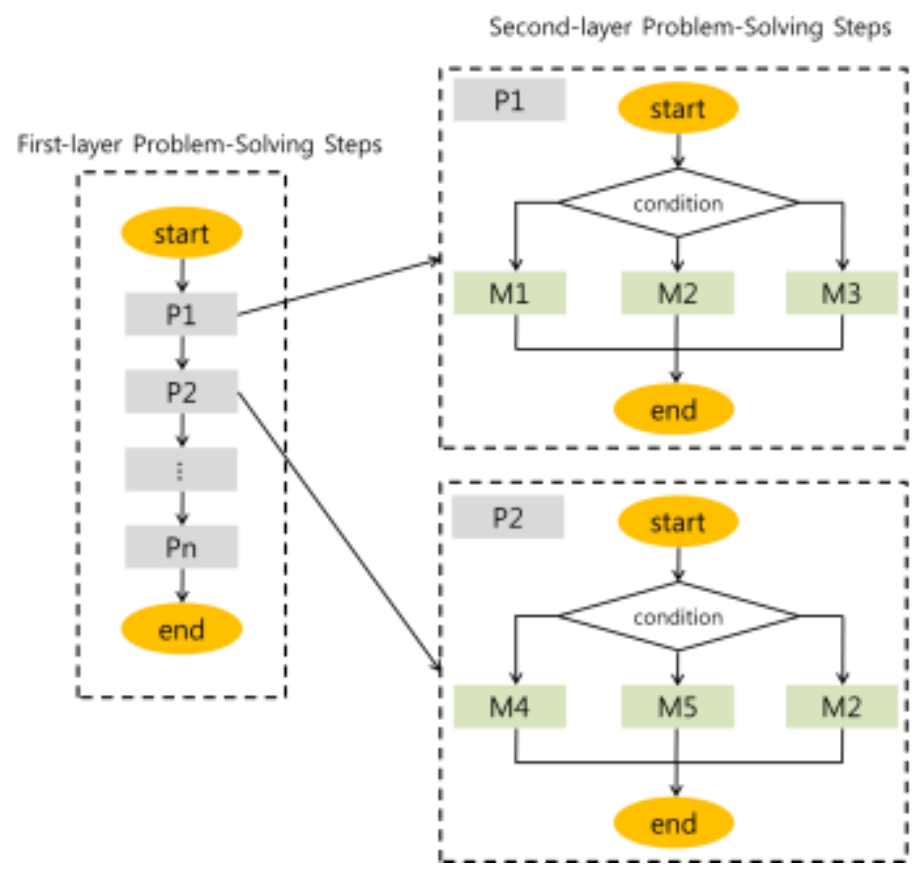

Fig. 3 Abstraction thinking model with 1st and 2nd layer problem-solving steps 


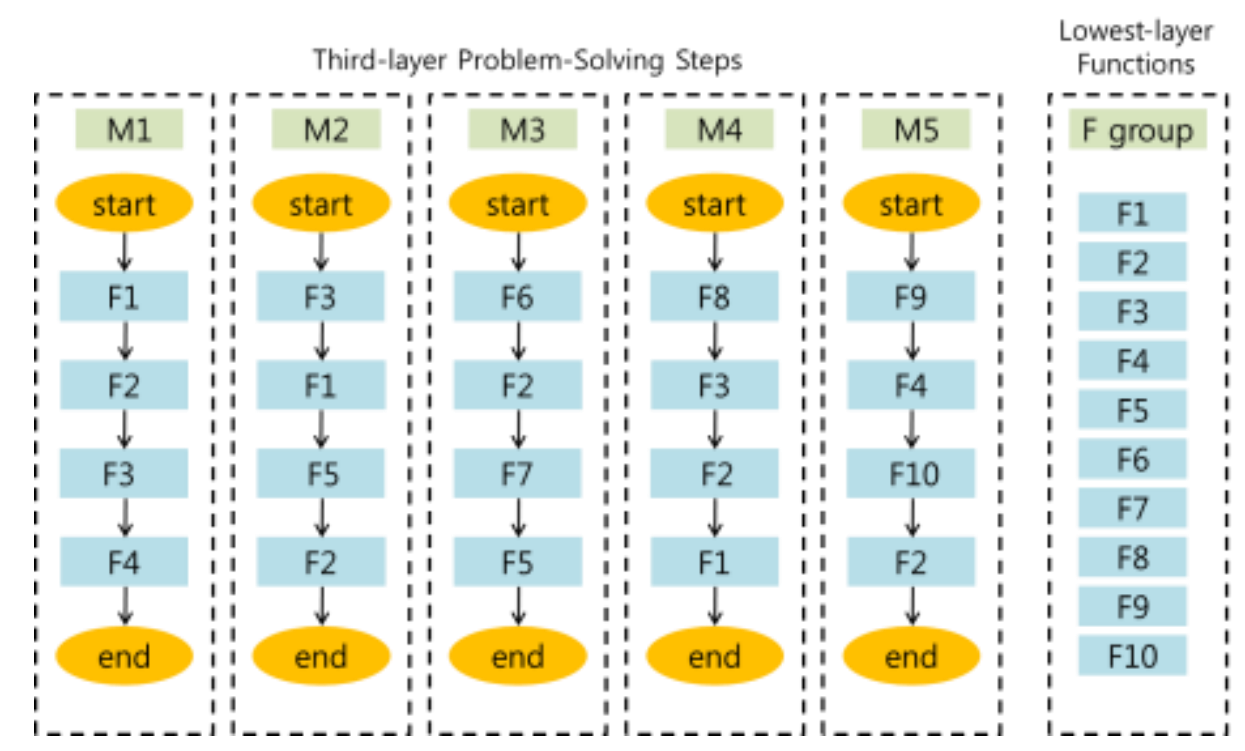

Fig. 4 Problem division and pattern recognition model with 3rd and lowest layer problem-solving steps

\section{SW-MAKING STEPS USING A CT MODEL FOR OX-TYPE SCORING PROGRAM DEVELOPMENT}

The second step is researching a problem-solving method. The detailed flow of the overall scoring program for several OX-type problems is shown in Figure 5. In Figure 5, a complex and large OX-type scoring main program is divided into three P1, P2, and P3 subprograms to solve small problems. And a large program collects small subprograms to solve a big problem. In Figure 6, there are three subprograms named as 'Ask for restart from the beginning', 'OX-type Q\&A', and 'View OX Overall score'. In the overall OXtype scoring program shown in Figure 7, one main program and three Pn subprograms are implemented separately based on the CT model.

The third step is designing algorithms for your computer to run. Algorithm denotes a detailed description of the procedure for resolving a problem based on a reference value that any computer recognizes [1-3]. In the fourth step, students create a program and run it. Students select Scratch 3.0 educational programming language (scratch.mit.edu). Educational programming languages (EPLs) provide immediate feedback to students' program results, enabling effective algorithm-making education for non-specialists [5].

In the example of OX-type scoring program, memory spaces to store three OX-type questions are named as 'OX-question \#1 OX-question \#3' variables. Memory space to store the overall score is named as 'OX-Overall score' variable. Score for each question is stored in a memory list array named as 'OX-Score list'. To search correct memory location and find correct score for each question, a variable named as 'Count' is used. This parameter set for the OX-type scoring program is shown in Figure 8. Three Pn subprograms are explained in the following subsections. 


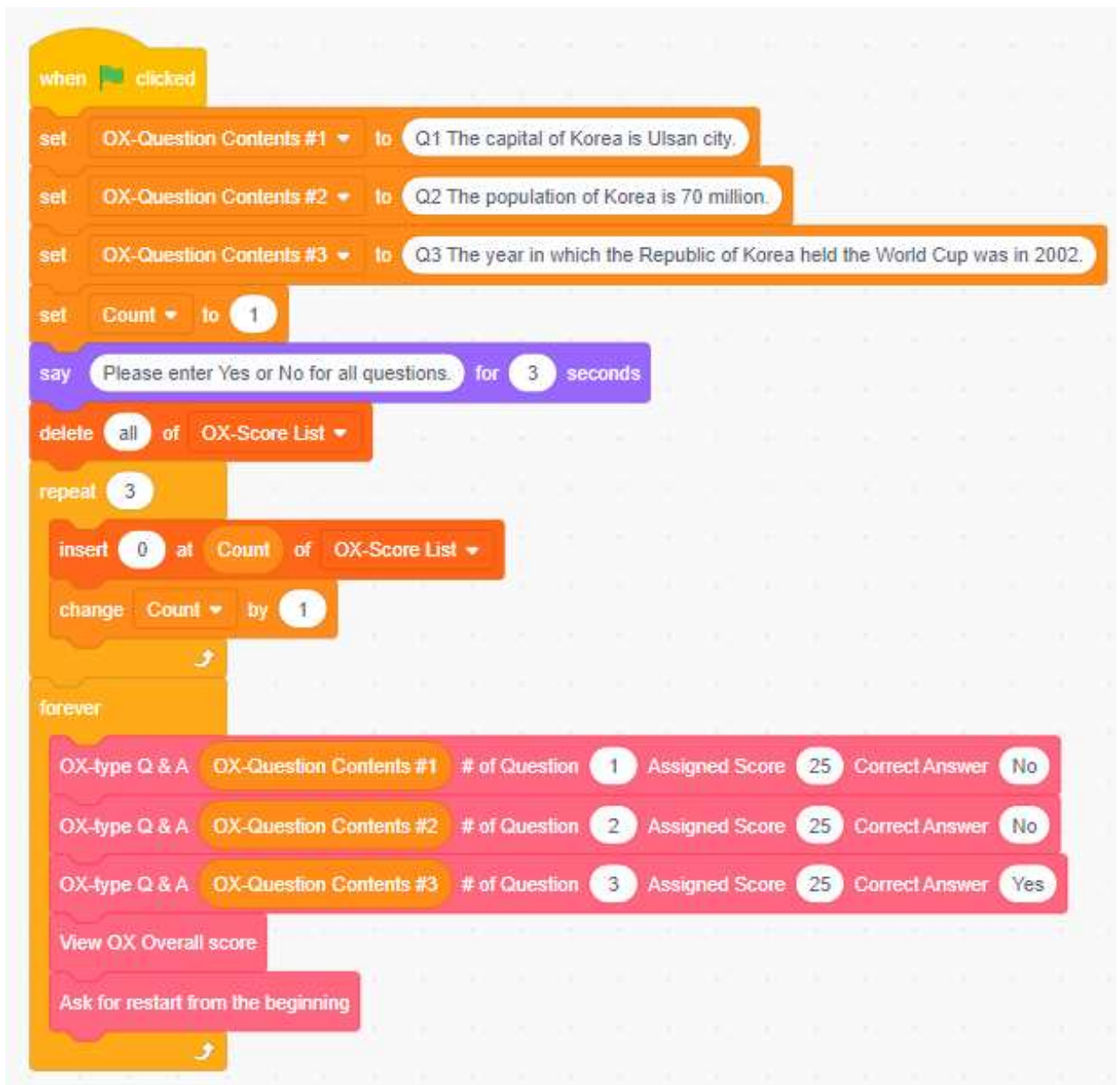

Fig. 5 Structure of OX-type scoring main program - 1st layer problem-solving steps

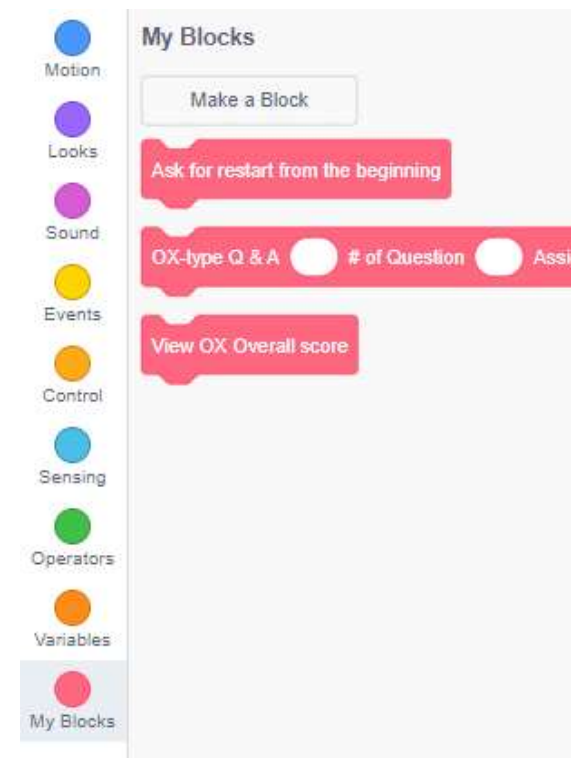

Fig. 6 P1, P2, P3 Subprograms in the main program 


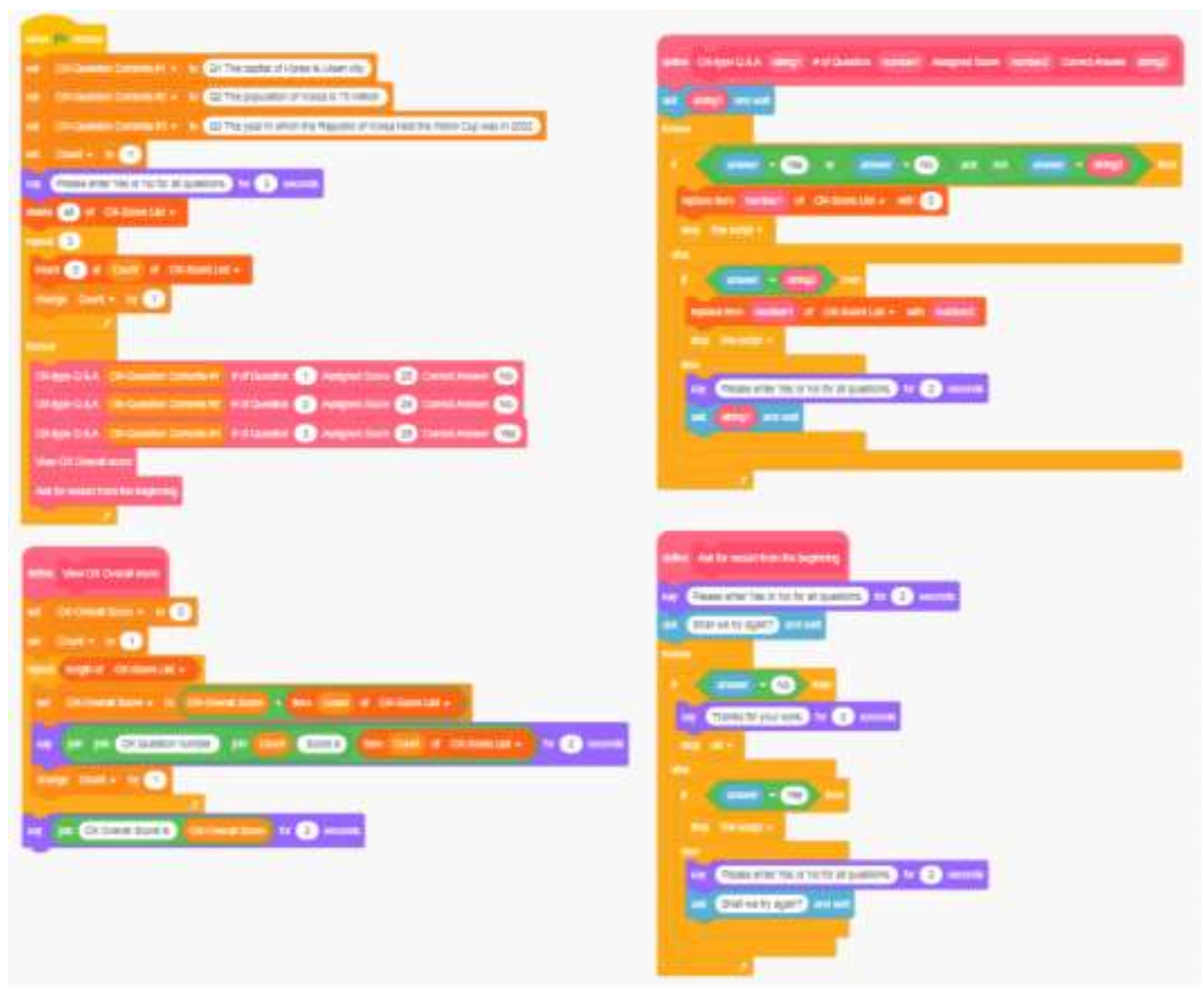

Fig. 7 CT-based Structure of overall OX-type scoring program

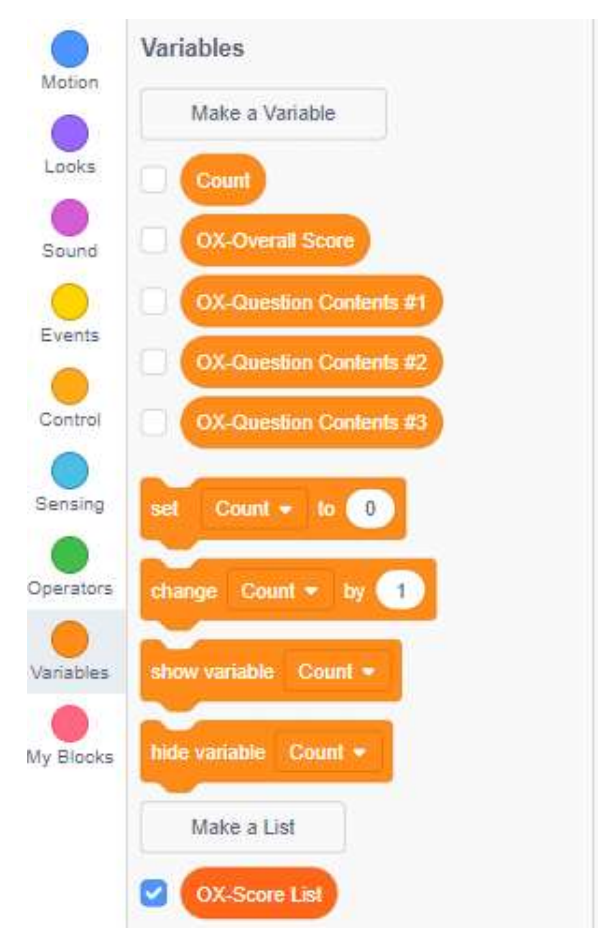

Fig. 8 Data structures with variables and list array in the program 


\subsection{P1 SUBPROGRAM - 'OX-TYPE Q\&A'}

The structure of 'OX-type Q\&A' subprogram is shown in Figure 9. It reads one of the three stored OX-type question contents from 'string 1' local variable and use it in place. This subprogram will know the correct answer from 'string 2' local variable. If the answer is available and equal to the 'string 2', the assigned score from 'number 2' local variable is stored at the location in the 'OX-Score list' that is pointed by the value in 'number 1' local variable. If the answer is available and unequal to the 'string 2', zero point is stored at the same location in the 'OX-Score list'. We can see that there exist several Mn and Fn blocks.

This subprogram takes into account the case where an unavailable answer is received instead of 'Yes' or 'No'. As a result of this infinite loop command block, whenever the unavailable answer case occurs, a guide message appears on the screen and computer waits an available answer as shown in Figure 10. On the contrary, whenever an available answer is received, the corresponding point is stored and a 'Stop this script' command is executed to escape the attached infinite loop block.

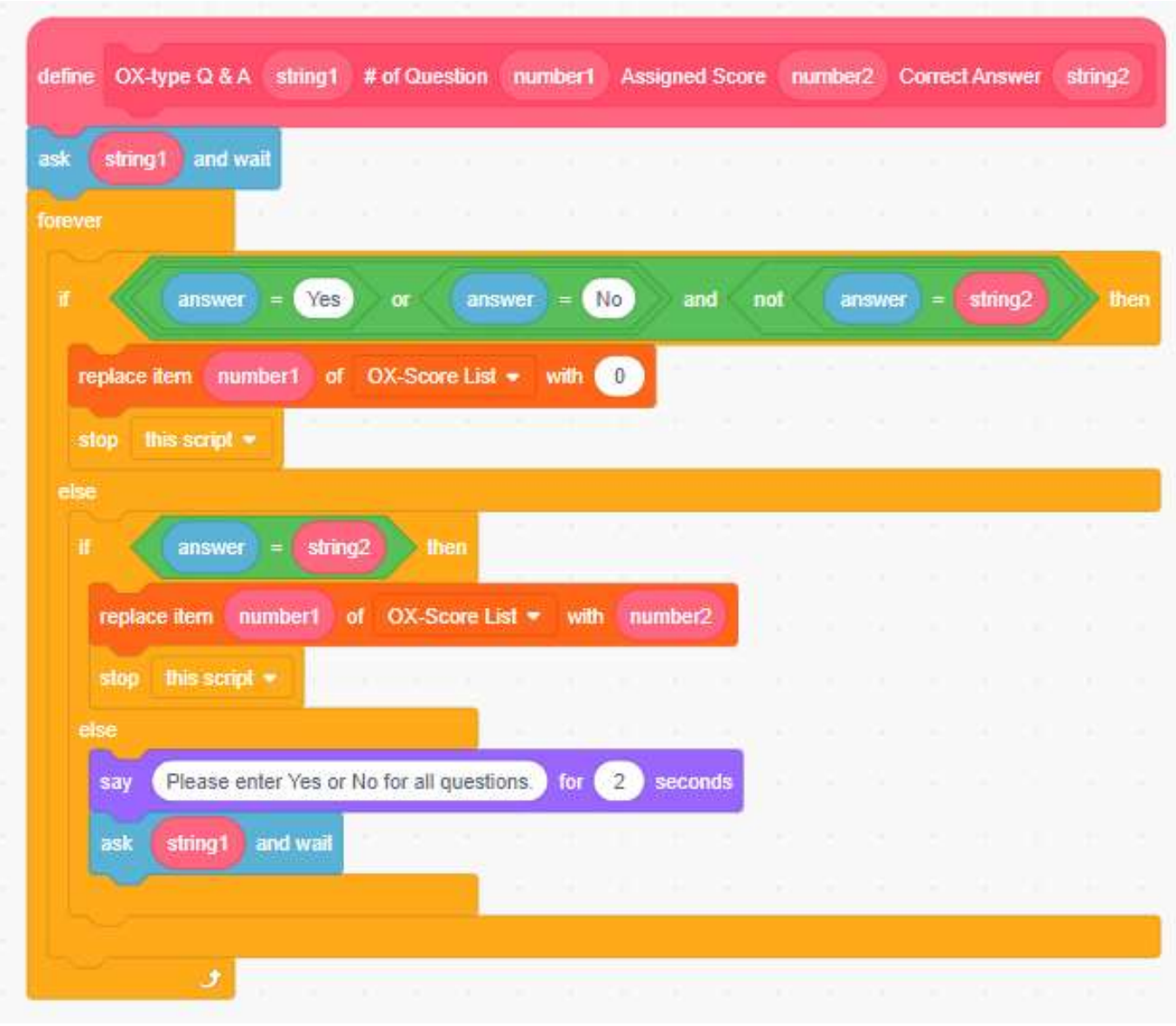

Fig. 9 Structure of 'OX-type Q\&A' subprogram 


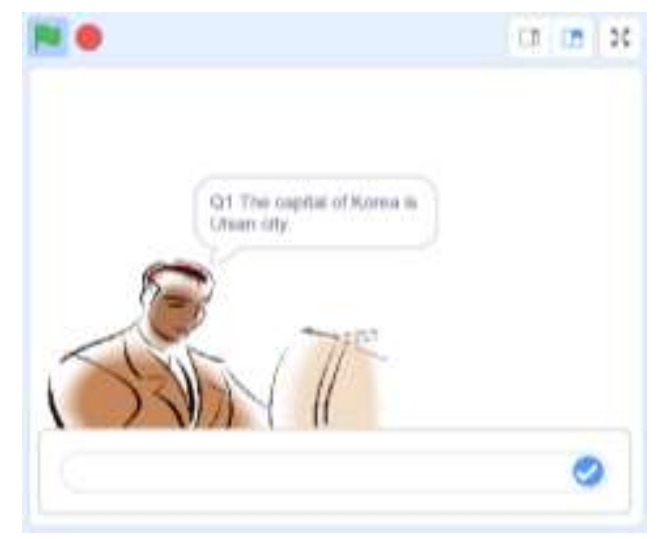

Fig. 10 Screen shot of 'OX-type Q\&A' subprogram play

\subsection{P2 SUBPROGRAM - 'VIEW OX OVERALL SCORE'}

The structure of 'View OX Overall score' subprogram is shown in Figure 11. It reads the three stored OX-type question scores from OX-Score list, one by one by using the 'Count' variable. Whenever it read one score from the OX-Score list, this score value is added to current value in the 'OX-Overall score' variable and stored. After that, each score is displayed one by one. Finally, 'OX-Overall score' value is shown at the screen. We can also see that there exist some Mn and Fn blocks in this subprogram.

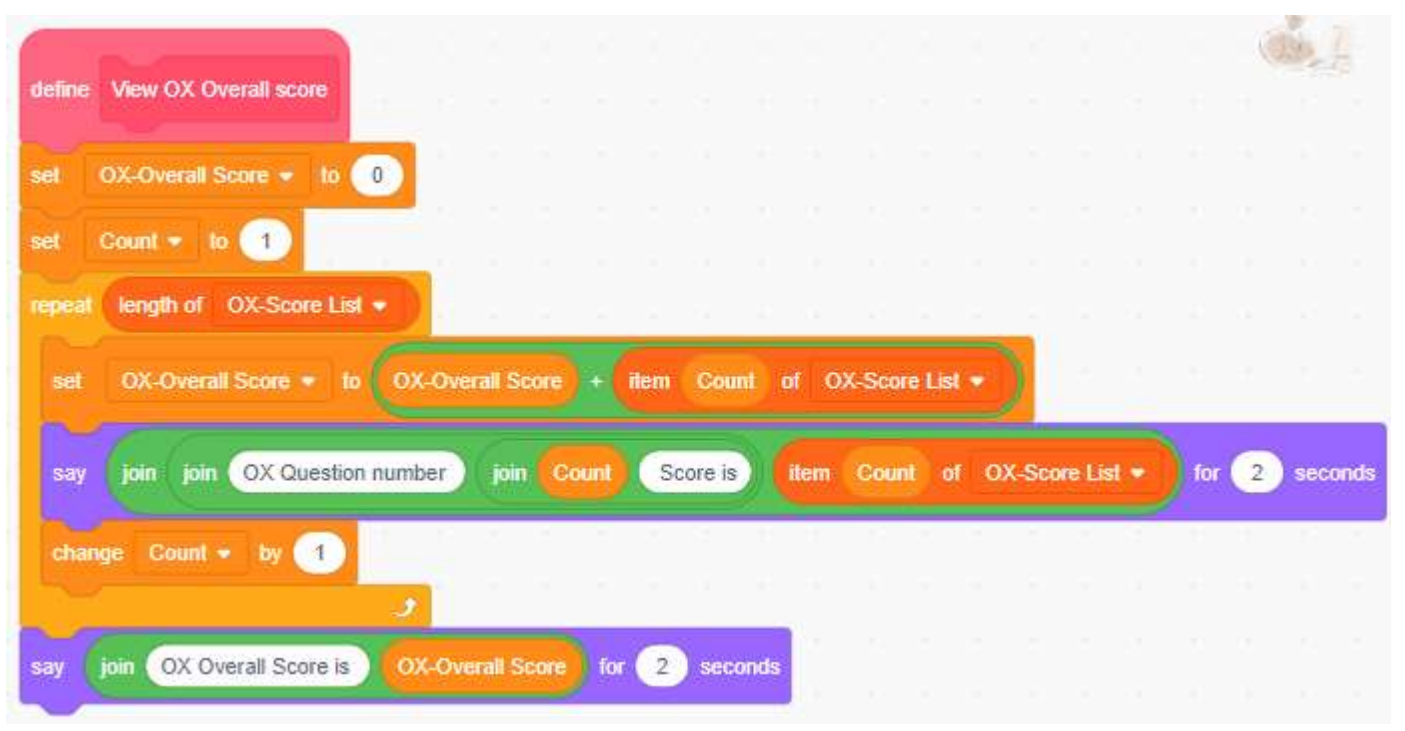

Fig. 11 Structure of 'View OX-Overall score' subprogram

\subsection{P3 SUBPROGRAM - 'ASK FOR RESTART FROM THE BEGINNING'}

The structure of 'Ask for restart from the beginning' subprogram is shown in Figure 12. This subprogram considers the case where an incorrect input, instead of 'Yes' or 'No', is entered. When an incorrect input occurs, a guide message appears on the screen and computer waits an available answer as shown in Figure 12. On the contrary, if an available input of 'Yes' occurs, 'Stop this script' Fn command is run to escape the infinite loop block in this subprogram and restart the 'OX-type Q\&A' subprograms in the main program.

On the other hand, if an available input of 'No' occurs, 'Stop all' command stops the entire OX-type scoring program. We can also see that there exist some Mn and Fn blocks in this subprogram. Students check results and correcting errors in their programs as the 
final step. They see the complete OX-type scoring program and understand the CT-based structure of the program as shown in Figure 7.

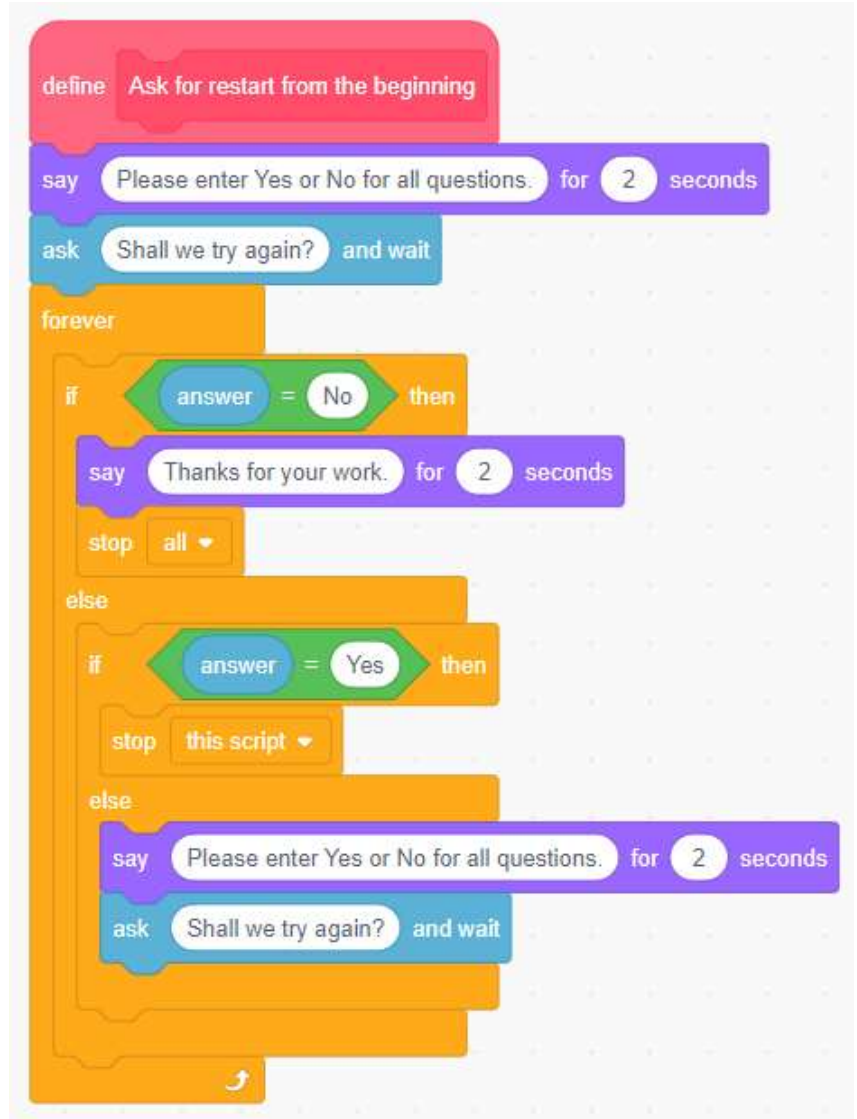

Fig. 12 Structure of 'Ask for restart from the beginning' subprogram

\subsection{DEVELOPMENT OF SCORING PROGRAM BY EACH TEAM}

The final step, required in the whole SW education stages of Figure 2, is composed as follows. After developing this OX-type scoring program, it is necessary to do Q\&A activity about results. Then, teacher provides experience that will appear when a part of the OX-type scoring program is modified. And teacher trains the ability to solve similar SW problems and apply to new SW problems. Teacher provides projects with various complexity according to the level of learners. Teacher provides an opportunity to complete a new project, either alone or in groups, from start to finish. If an error occurs, teacher let the learners solve it by themselves and answers their questions.

\section{CONCLUSION}

In this paper, a model of CT processes is proposed to achieve the goals of SW education. Also, as its use-case in the SW education, an OX-type scoring program is developed with scratch 3.0 for non-major students' project assignment. However, in implementing with scratch 3.0 software, if the students are familiar with the function block and usage of each command block, the project will proceed easily. Otherwise, students cannot understand easily the difference between their algorithms and the implemented real programs. Students can understand how to use control blocks such as iterations, conditions and logic operations, through this SW development class. Furthermore, this proposal can be applied in various SW education classes to teach the problem-solving and SW-making steps using the CT model. 


\section{Author Contributions}

Kyeong Hur wrote the manuscript. Won-Sung Sohn designed experiments. Kil Young Kwon provided technical supports.

\section{References}

[1] J. M. Wing, "Computational Thinking”, Communications of the ACM, vol. 49, no. 3, (2006), pp. 33-35.

[2] J. M. Wing, "Computational thinking and thinking about computing," Philosophical transactions of the royal society of London A: mathematical, physical and engineering sciences, vol. 366, no. 1881, (2008), pp. 3717-3725.

[3] S. Yang, "Necessity of Computational Thinking. Korea Information Processing Society Magazine," vol. 24, no. 2, (2017), pp. 4-12.

[4] X. Wang and H.-C. Kim, "Text Categorization with Improved Deep Learning Methods", Journal of information and communication convergence engineering, vol. 16, no. 2, June (2018), pp. 106-113.

[5] D.J. Malan and H.H. Leitner, "Scratch for budding computer scientists," SIGCSE Bulletin, vol.39, no.1, (2007), pp.223-227.

[6] J. Li, S.Y. Shin and H.C. Lee, "Text Mining and Visualization of Papers Reviews Using R Language", Journal of Information and Communication Convergence Engineering, vol. 15, no. 3, September (2017), pp. 170-174.

[7] A. Elzamly, B. Hussin, S. S. A. Naser and Mohamed Doheir, "Classification of Software Risks with Discriminant Analysis Techniques in Software planning Development Process", International Journal of Advanced Science and Technology, vol. 81, August (2015), pp. 35-48.

[8] G. Chandrika, "Study on Software Reliability and Reliability Testing", Asia-pacific Journal of Convergent Research Interchange, vol. 1, no. 1, March (2015), pp. 7-20.

[9] S. H. Sung and P. Z. Khan, "Quantitative and Qualitative Approach for IT Risk Assessment", Asiapacific Journal of Convergent Research Interchange, vol. 1, no. 1, March (2015), pp. 29-35.

[10] S. M. Shin and Sk. Uroosa, "Predicting Software Reliability Using Particle SWARM Optimization Technique", Asia-pacific Journal of Convergent Research Interchange, vol. 1, no. 3, September (2015), pp. 17-30.

[11] M. S. Srinivas and G. Pradeepini, "Feature Selection based Neural Networks for Software Defect Prediction", International Journal of Security Technology for Smart Device (IJSTSD), vol. 1, no. 1, April (2014), pp. 7-12.

[12] J. Tae, "Development of STEAM Education Program Utilizing IoT Teaching Aid for Middle School Students and Its Application", International Journal of Computer Science and Information Technology for Education (IJCSITE), vol. 2, no. 1, May (2017), pp. 15-20.

[13] J.-H. Eom, "Problems and Improvement of the Curriculum for Effective Cyber Security Education and Training”, Journal of Security Engineering, vol. 12, no. 4, (2015), pp. 337-350. 